Published by Al-Nahrain College of Medicine P-ISSN 1681-6579

E-ISSN 2224-4719

Email: iraqijms@colmed-alnahrain.edu.iq

http://www.colmed-alnahrain.edu.iq

http://www.iraqijms.net

Iraqi JMS 2019; Vol. 17(2)

\title{
The Effects of Gender and Hand Dominancy on Motor Unit Number Estimation in A Sample of Healthy Iraqis Using Two Different Methods
}

\author{
Safa Dh. Abdul Muneem ${ }^{1}$ MSc, Hussein G. Kaddori ${ }^{2} P h D$ \\ ${ }^{1}$ Section of Neurophysiology, Al-Imamein Al-Kadhimein Medical City, Baghdad, Iraq, ${ }^{2}$ Dept. of Physiology, College of \\ Medicine, Al-Nahrain University, Baghdad, Iraq
}

\begin{abstract}
Background Motor unit number estimation (MUNE) is a unique electrophysiological technique that was developed to determine a numeric estimate of the number of innervating axons. This technique can be used to determine the approximate number of motor neurons in a muscle or group of muscles.

Objective To test the MUNE values according to subject's gender and hand dominancy in the normal population.

Methods Healthy volunteers who had neither neuromuscular nor systemic/ metabolic disease, with normal neurological examination were studied. Ninety hands of 56 healthy volunteers ( 11 males and 45 females) with ages ranging from (24-58) years were included in the study. All had normal median nerve conduction studies. Manual incremental (INC) method and adapted multiple points stimulation (AMPS) method were performed for MUNE.

Results Gender did not have an effect on the scores according to the two studied methods ( $p=0.054$ by INC method and $p=0.700$ by AMPS method). Hand dominancy also show no statistically significant difference of the scores of MUNE according to both studied methods ( $p=0.091$ ) by INC method and $(p=0.051)$ by AMPS method.

Conclusion Incremental stimulation and adapted multiple point stimulation are reliable and easily applicable methods with same reproducibility in estimating motor units with no significant effect for subject gender or hand dominancy.

Keywords Motor unit, motor unit number estimation (MUNE), incremental stimulation (INC) method, adapted multiple point stimulation (AMPS) method

Citation Abdul Muneem SD, Kaddori HG. The effects of gender and hand dominancy on motor unit number estimation in a sample of healthy Iraqis using two different methods. Iraqi JMS. 2019; 17(2): 153-160. doi: 10.22578/IJMS.17.2.9
\end{abstract}

List of abbreviations: AMPS = Adapted multiple point stimulation, $\mathrm{APB}=$ Abductor pollicis brevis muscle, $\mathrm{CMAP}=$ Compound motor action potential, $\mathrm{CTS}=$ Carpal tunnel syndrome, $\mathrm{EMG}=$ Electromyography, $E P=$ Evoked potentials, $I N C=$ Incremental stimulation, $\mathrm{MCP}=$ Metacarpophalangeal joint, $\mathrm{MNCS}=$ Motor nerve conduction study, $\mathrm{MU}=$ Motor unit, $\mathrm{MUNE}=$ Motor unit number estimation, SNCS = Sensory nerve conduction study

\section{Introduction}

A motor unit (MU) is defined as a single anterior horn cell or brain stem motor neuron, its peripheral axon (which travels in a cranial or peripheral nerve), and each of the muscle fibers innervated by that axon. they are considered as the final common pathway of the motor system (1).

The numeric determination of numbers of MUs is described as motor unit number estimation (MUNE) (2). MUNE provides determination of functional MU numbers quantitatively as the 
most parallel to the real numbers and can be performed in various techniques ${ }^{(1)}$.

The thought of estimating MUs was firstly mentioned by McComas in 1967. Estimating the functional MUN in a human muscle group in vivo has been facilitated by using computeraided MUNE techniques. There have been at least 10 different MUNE techniques reported up till now and each of which has some advantages and disadvantages. There have not been so many studies directly quantifying the effect of hand dominancy or even the effect of gender on MUNEs although these electrophysiological techniques have been available ${ }^{(2-5)}$.

This study aimed to determine the effect of subject gender and hand dominancy on estimated MUN in Abductor pollicis brevis (APB) muscle of healthy subjects using incremental stimulation (INC) and adapted multiple point stimulation (AMPS) methods

\section{Methods}

A cross sectional study was conducted at the Neurophysiology Unit at Al-Imamein AlKadhimein Medical City during a 4-month period (Nov. 2017 to Mar. 2018). The study was approved by the Institute Review Board of the College of Medicine, Al-Nahrain University. An ethical consent was taken from each participant to be enrolled in the study.

\section{Subjects}

The study was performed on 90 hands of 56 healthy volunteers with a mean age of $36.22 \pm 7.32$ years (age range $=24-58$ years) . Eleven subjects were males (19.6\%) and 45 were females (80.4\%). Forty-nine subject $(87.5 \%)$ were right-handed and only seven were left-handed (12.5\%). The study excludes any subject with history of wrist fracture or surgery, carpal tunnel syndrome, cervical radiculopathy, hereditary or acquired peripheral neuropathy.

Healthy volunteers with neither Neuromuscular disease nor any systemic/metabolic disease were included in the study. All subjects were considered to be moderately active for their respective age groups. Before the study, systemic and neurological examinations of subjects were made and if they had normal findings, conventional neurophysiological studies of median and ulnar nerves were performed and if these were also normal, MUNE studies by INC stimulation and AMPS techniques were carried on.

\section{Instrumentation}

The following were used for all electrodiagnostic testing:

Computerized Electromyography/ Evoked potentials (EMG/EP) machine (Cadwell, 8channel electromyograph) supplemented with different types of electrodes including grounding electrode used to protect the subject against electrical hazard and to reduce stimulus artifacts and interference, stimulating surface electrodes was used to stimulate the nerves through the skin and surface recording electrodes.

\section{Electrophysiological studies}

For each subject, conventional neurophysiological studies (sensory, motor and $F$ wave studies) were performed to exclude peripheral neuropathy. MUNE of both APB muscles using both INC \& AMPS methods were performed.

\section{Sensory nerve conduction study}

An antidromic method was used for sensory nerve conduction study (SNCS) determination, in which, the nerve was proximally stimulated from the trunk and the evoked activity was distally recorded from a finger. The parameters studied were the sensory latency (SL), sensory nerve action potential (SNAP) amplitude from peak to peak and sensory nerve conduction velocity (SNCV) measured by dividing the conduction distance (d) by the SL and measured in meter/second $(\mathrm{m} / \mathrm{sec})^{(6)}$.

\section{Motor nerve conduction study and F-wave}

The motor nerve was simulated at two points along its course, by applying stimuli at the 
distal and the proximal sites of the nerve and recording from the muscle innervated by that nerve. The parameters studied were distal motor latency (DML). Motor nerve conduction velocity (MNCV) measured by dividing the distance between the two stimulation points over the difference between the latencies of the recorded responses ensuring both compound muscle AP (CMAP) configurations must be similar in addition to $F$ wave latency measured from the stimulus artifact to the beginning of the evoked potential ${ }^{(6)}$.

\section{Motor-unit number estimation}

MUNE values are calculated from the ratio:

\section{maximal compound muscle action potential (CMAP) amplitude or area}

\section{average single motor unit potential (MUP) amplitude or area}

MUNE test was performed using a manual INC method. MUNE was recorded from a surfaceactive recording electrode placed over the motor point of the APB with the reference over the metacarpal phalangeal (MCP) joint of the thumb. The median nerve was stimulated at wrist by Cadwell electrical stimulator, at $8 \mathrm{~cm}$ proximal to the active electrode. For accurate nerve stimulation, the exact site of stimulation was marked by pin at wrist prior to stimulation. A built-in MUNE analysis program in the Cadwell EMG device was used, and the maximum $M$ response was first obtained by increasing stimulation intensity and a maximal CMAP is recorded. Next, the display sensitivity is raised to $100-200 \mu \mathrm{V} /$ div to help visualize low amplitude steps in the response envelope. The stimulus intensity is lowered to 3-10 mA in order to activate the first axon, indicated by an all-or-none response. By small increases in stimulation intensity, an envelope of responses is obtained with 8-10 discrete steps before the increments in the envelope become indistinguishable. The number of steps is divided into the peak to-peak amplitude of the envelope to determine the average amplitude of each step.

This average value represents the average single MUP (S-MUP), and is used to calculate the MUNE value (figures 1 and 2) ${ }^{(7)}$.

\section{Adapted multiple point stimulation (AMPS) method}

Multipoint incremental MUNE method with the Shefner modification is a noninvasive, easy to perform method with high reproducibility $(7,8)$.

By stimulating the nerve at many sites, this technique yield data from MUs with different morphologies. The recording electrode was placed on the belly of the APB muscle and a maximum CMAP was obtained by applying a supramaximal stimulus.

Median nerve of the right and left hand was studied. Recording electrodes were placed on the median nerve innervated APB muscle, using the standard belly tendon method. The MUNE program recorded the maximum CMAP amplitude obtained. Then display sensitivity was raised to $100-200 \mu \mathrm{V} /$ div to help visualize low amplitude steps in the response envelop and the stimulus intensity was lowered to 3-10 $\mathrm{mA}$ in order to activate the first axon, indicated by an all-or-none response. Again, by small increase in stimulation intensity, an envelope of responses is obtained with 3-5 discrete steps before jumping to the next site. 


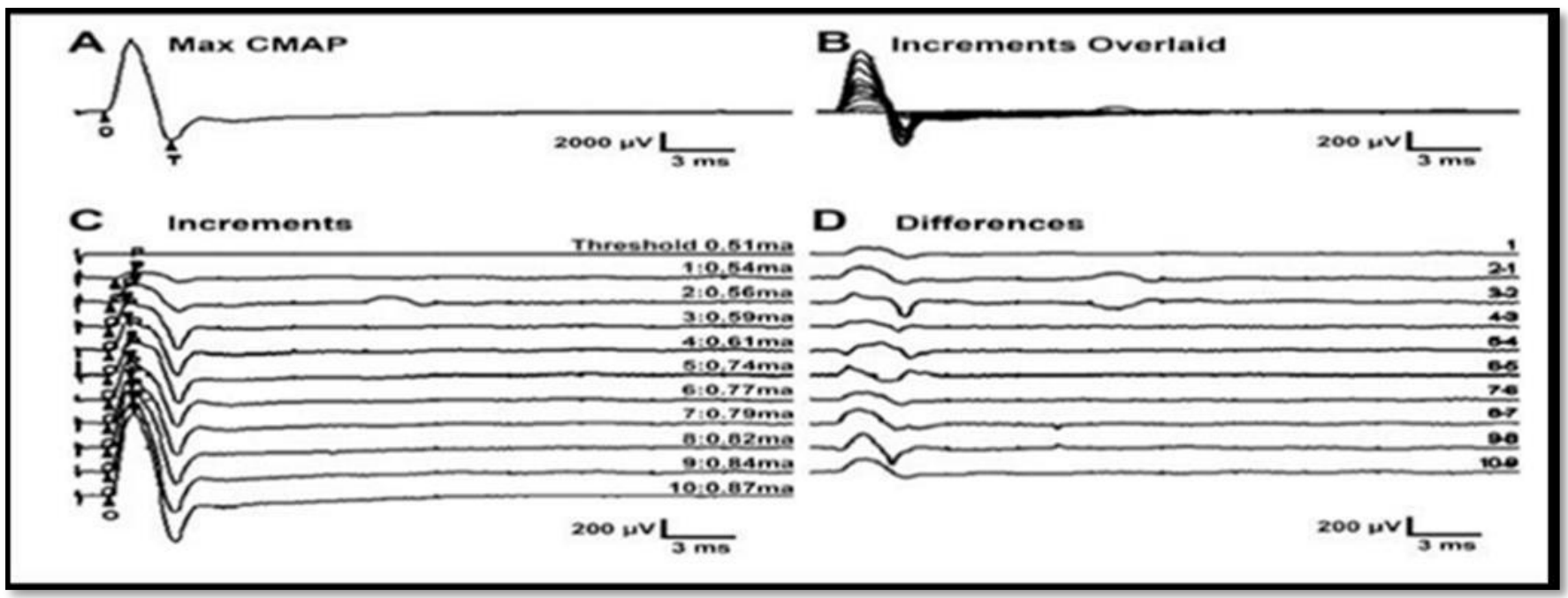

Figure 1. Scheme of incremental stimulation. A. with maximal compound muscle action potential, CMAP max. B. 10 stacked incremental evoked potentials. C. Individual evoked potentials and $D$. single motor unit potentials, SMUPs ${ }^{(6)}$

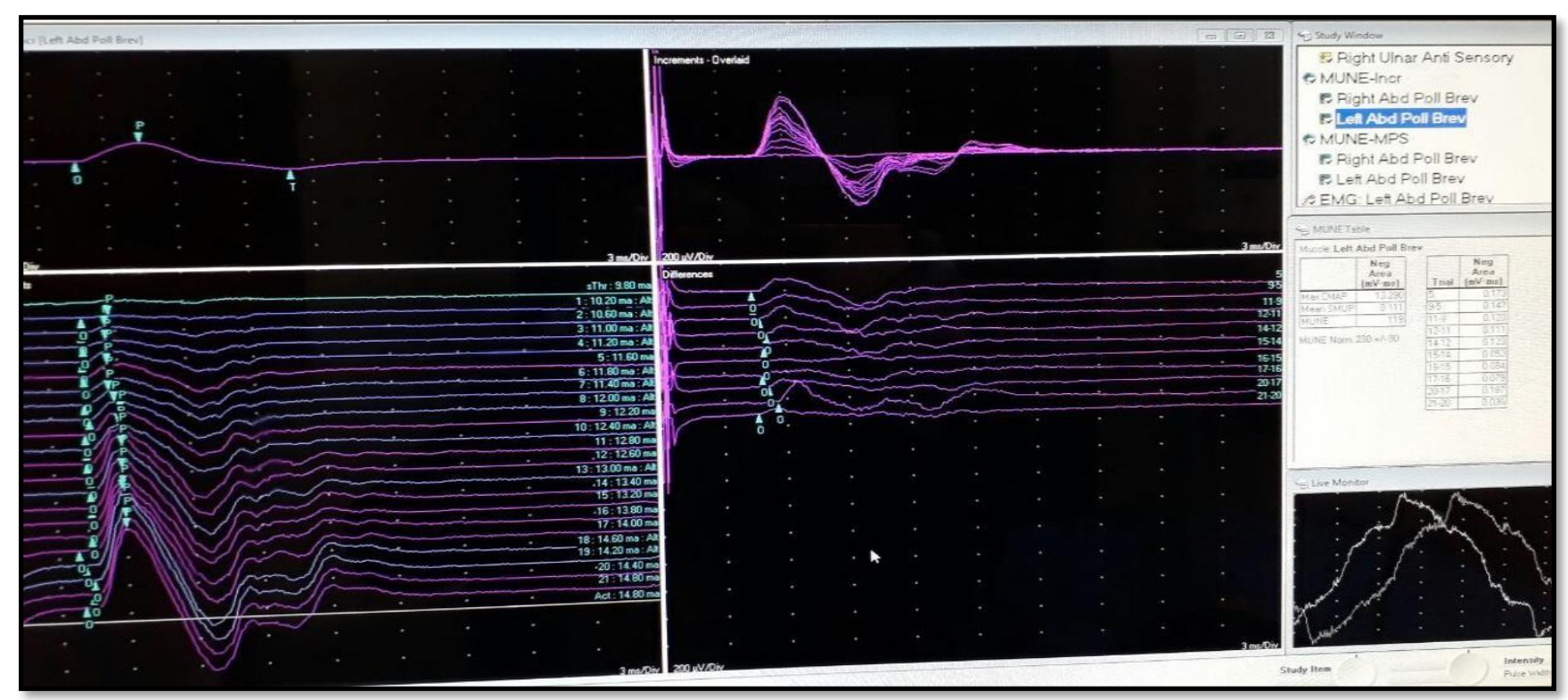

Figure 2. A photographic picture. of Cadwell EMG screen during incremental MUNE study

Using standard 3-site motor conduction program traces were obtained and superimposed. Three stimulus locations were used for the median nerve; $2 \mathrm{~cm}$ proximal to the wrist crease, $4 \mathrm{~cm}$ proximal to the first stimulation site, and in the cubital fossa. Optimum stimulus location was determined using a submaximal stimulus and moving the stimulator to evoke the greatest response.
Three responses were obtained at each stimulation site with each response of $25 \mu \mathrm{V}$ incremental amplitude. The negative peak amplitude of the third response was recorded. Stimulation at the second and third location was identical to the first location. Once the sample collection was complete, we reviewed all tracings for potential repeating MUs (so that they were not included more than once). Using 
standard 3-site motor conduction program traces were obtained and superimposed (figure
3) (5) The test last about 30 minutes for each subject.

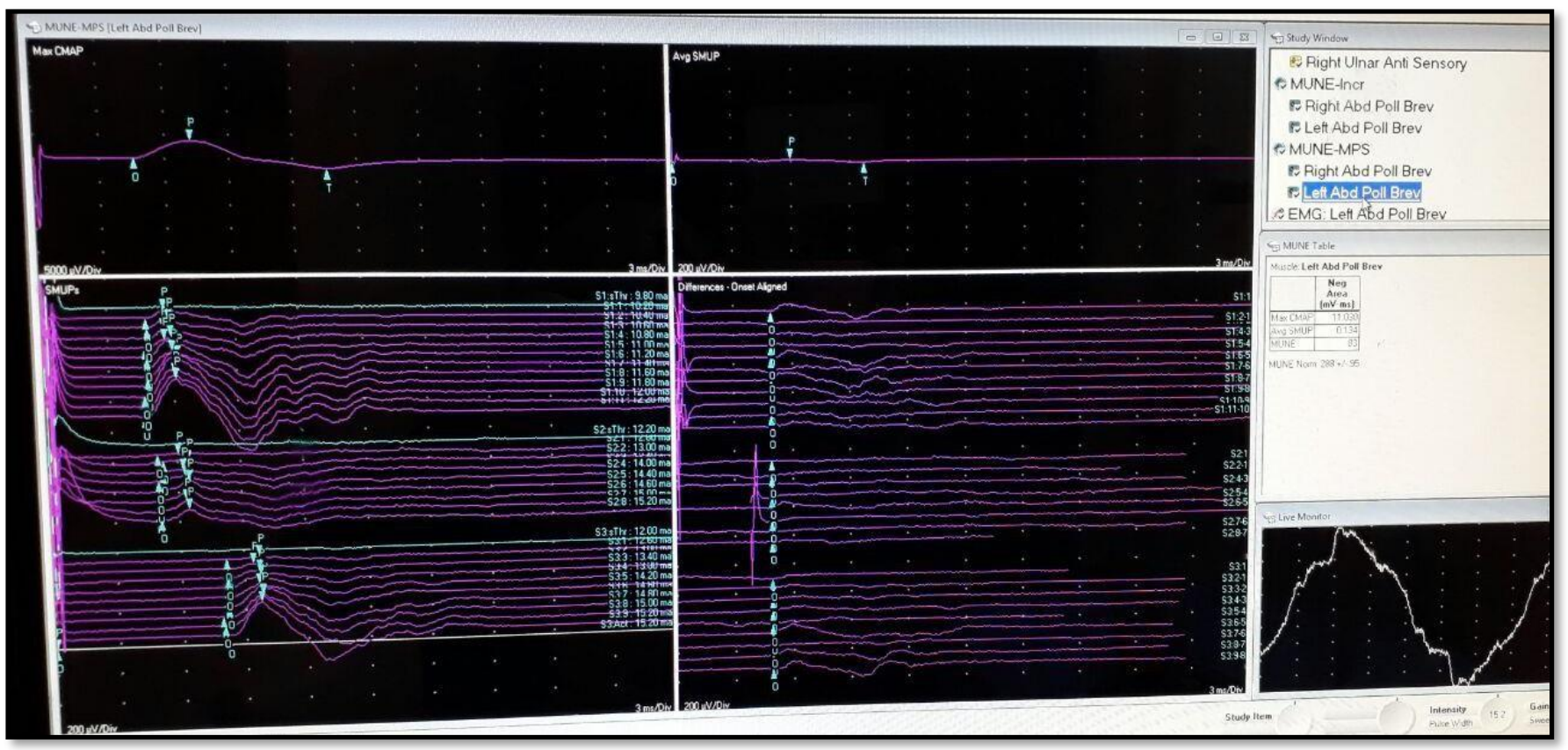

Figure 3. Photographic picture. of Cadwell EMG device during Adapted multiple point stimulation MUNE study

\section{Statistical analysis}

The statistical analysis was obtained using statistical package of social sciences (SPSS) version 23 software and Microsoft Office Excel 2016.

All data were expressed as mean \pm SD. Paired $t$ test was used to compare the dominant and non-dominant side and between males and females. P-value of 0.05 or less was considered significant.

\section{Results}

This study found that MUNE values of APB muscles by both techniques are higher in male group than in female (but not statistically significant).

Considering the hand dominancy, we found that the non-dominant hand APB muscle contains higher MUNE value that is $214.74 \pm 50.18$ by INC. method and $272.35 \pm 68.49$ by AMPS method for nondominant APB and $196.18 \pm 49.2$ by INC. method and $244.82 \pm 54.27$ by AMPS method for dominant APB muscle- as shown in tables 1 $\& 2$. 
Table 1. MUNE value of the APB muscles in female and male subjects by INC and AMPS methods (unpaired $t$ test)

\begin{tabular}{cccc}
\hline MUNE method & $\begin{array}{c}\text { Females } \mathbf{N}=67 \\
\text { Mean } \pm \text { SD }\end{array}$ & $\begin{array}{c}\text { Males } \mathbf{N}=\mathbf{2 3} \\
\text { Mean } \pm \text { SD }\end{array}$ & P value \\
\hline INC method & $197.38 \pm 47.15$ & $226.44 \pm 56.11$ & 0.054 \\
AMPS method & $254.11 \pm 63.58$ & $259.67 \pm 51.69$ & 0.700 \\
\hline
\end{tabular}

MUNE = motor unit number estimation, INC= incremental stimulation method, AMPS = Adapted multiple point stimulations method, $\pm \mathrm{SD}=$ standard deviation.

Table 2. MUNE value of APB muscles of the dominant and non-dominant hands (unpaired $t$ test)

\begin{tabular}{|c|c|c|c|}
\hline MUNE method & $\begin{array}{c}\text { Dominant hand } \mathrm{N}=56 \\
\text { Mean } \pm S D\end{array}$ & $\begin{array}{c}\text { Non-dominant hand } \mathrm{N}=34 \\
\text { Mean } \pm \text { SD }\end{array}$ & $P$ value \\
\hline INC method & $196.18 \pm 49.2$ & $214.74 \pm 50.18$ & 0.091 \\
\hline AMPS method & $244.82 \pm 54.27$ & $272.35 \pm 68.49$ & 0.051 \\
\hline
\end{tabular}

MUNE = motor unit number estimation, INC= incremental stimulation method, AMPS = Adapted multiple point stimulations method, $\pm \mathrm{SD}=$ standard deviation.

\section{Correlation of two MUNE methods}

The above data denotes - no significant deference in MUNE values between INC and AMPS techniques. As seen in figure 4, a strong - positive correlation between values obtained by both methods was found $(r=0.769$ and $p<$ 0.001).

\section{MUNE value}

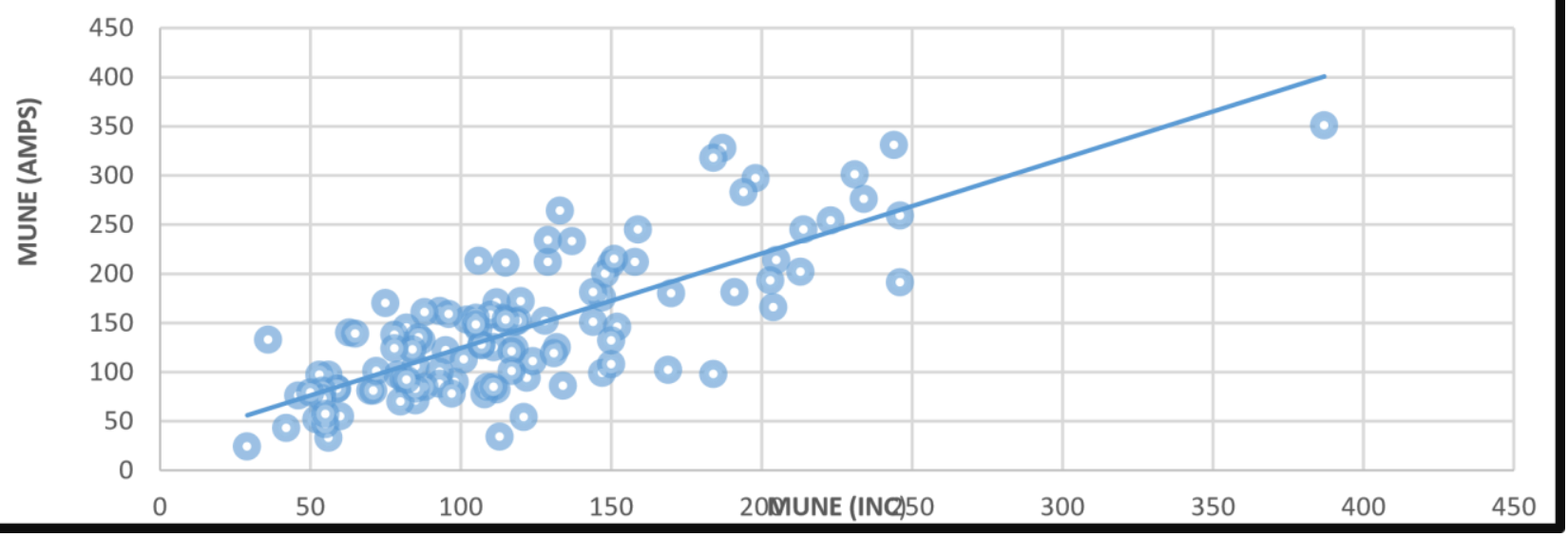

Figure 4. Correlation of INC and AMPS methods for MUNE

\section{Discussion}

For now, there have been many available MUNE techniques share in common studying the features of MUs in an attempt to determine quantitively the functional MUN - as the most similar to the real numbers. All the

\section{8}


techniques have been developed based on manual incremental method ${ }^{(9)}$.

The aim of MUNE methods is to calculate the number of fibers in a muscle almost correctly and relative to the real numbers. Certainly, the most accurate way of this is to estimate fibers histologically. Studies dealt with correlation between histological and electrophysiological studies are scarce ${ }^{(9,10)}$. In general, according to the test reliability, the necessary period for completing study, easiness, obtaining data parallel to disease and sufficiency in determining progression of disease, none of these different methods is superior to each other ${ }^{(11)}$.

Manual INC and AMPS methods were used in this study. Results of the study revealed quite similar values in standard conditions between the two methods. although practically we prefer to use incremental stimulation method since it easier to stimulate median nerve at wrist in comparison with three stimulation sites for median nerve in AMPS.

Furthermore, the study demonstrated that gender has no significant effect on MUNE values (though the results show that MUNE in males higher than that of females but statistically not significant). This may be explained by the more muscular components of male body or hormonal differences, increasing sample size is mandatory to study this difference. These results are similar to that reported by Yerdelen et al. in 2006 who state that gender had no effect on MUNE value although his results show higher values in male than females but statistically not significant ${ }^{(1)}$.

Also, we found that MUNE values of median innervated APB muscle by both techniques are not statistically significantly different according to hand dominancy (even though the results show higher values in non-dominant hands but still not significant). The current data were comparable with those reported by $\mathrm{Li}$ et al. who state that there was no statistically significant difference between both dominant and non-dominant hand regarding first dorsal interossi (FDI) and thenar muscles regardless his results show high motor unit number index
(MUNIX), lower motor unit size index MUSIX in non-dominant hands for both muscles $(12,13)$.

Handedness is associated with brain lateralization. Asymmetrical excitability in the corticospinal system has been observed in many researches with transcranial magnetic stimulation ${ }^{(14,15)}$. Contradictory evidence was also reported showing no significant influence of handedness on motor evoked potentials (16). The neural mechanisms underlying handedness is not fully understood yet. Regardless of neural origins of handedness, this study examined the MUNE estimations in dominant and non-dominant hand muscles. The MUNE values from bilateral hand muscles were within the range of previously reported reference values $(3,13,17)$. No significant difference in MUNE values was observed between the dominant and non-dominant hands for the thenar muscles, implying that the population of MUs or spinal motor neurons may not be associated with handedness ${ }^{(13)}$.

In contrast to investigations on the lateral asymmetry in the central nervous system, aspects related to lateral asymmetry of the peripheral nervous system have been relatively less studied. By applying the MUNE technique, a previous study observed that the number of motor units of the APB muscle was higher for the non-dominant hand than the dominant hand muscles ${ }^{(18)}$. So higher sample size study is recommended to clarify this association.

The non-significant difference in MUNE results obtained by INC and AMPS methods in this study denotes that both are similarly reproducible and are equally effective and none of them was superior to the other which is in accordance with the other researchers as $\mathrm{Xu}$ et al. who stated that both MUNE methods are similarly reproducible and are equally effective in in estimating motor units and their reduction with aging and ALS patients (17).

In conclusion the present study revealed that the gender of subjects or hand dominancy did not affect MUNE value, both INC and AMPS techniques are easily applicable, similarly reproducible and are equally effective MUNE methods. 


\section{Acknowledgement}

Authors would like to thanks to the all members of Neurophysiology Unit in AlImamein Al- Kadhimein Medical City for their help.

\section{Authors contribution}

Dr. Abdul Muneem: collection of data, analysis of them. Interpretation and discussion of results done by both authors.

\section{Conflict of interest}

The authors declare no conflict of interest.

\section{Funding}

None.

\section{References}

1. Yerdelen D, Koç F, Sarica Y. The effects of gender and age on motor unit number estimation in a normal population. Acta Neurol Belg. 2006; 106(1): 5-8.

2. Daube JR. Estimating the number of motor units in a muscle. J Clin Neurophysiol. 1995; 12(6): 585-94.

3. Tan U. Lateralization of the Hoffmann reflex from the long flexor thumb muscle in right- and left-handed normal subjects. Int J Neurosci. 1989; 48(3-4): 313-5.

4. Preston DC, Shapiro BE. Electromyography in neuromuscular disorders. $3^{\text {rd }}$ ed. ClinicalElectrophysiologic Correlations Elsevier. 2013; Chapter 17, p. 275.

5. Gooch CL, Doherty TJ, Chan KM, et al. Motor unit number estimation: a technology and literature review. Muscle Nerve. 2014; 50(6): 884-93. doi: 10.1002/mus.24442.

6. Vasquez L. Development of a motor unit number estimation technique in normal dogs: a potential biomarker for canine degenerative myelopathy. MSc thesis in Veterinary Biomedical Sciences. University of Missouri-Columbia; 2011.

7. Shefner JM, Cudkowicz ME, Brown RH Jr. Comparison of incremental with multipoint MUNE methods in transgenic ALS mice. Muscle Nerve. 2002; 25(1): 3942.

8. Gawel M, Kostera-Pruszczyk A. Effect of age and gender on the number of motor units in healthy subjects estimated by the multipoint incremental MUNE method. J Clin Neurophysiol. 2014; 31(3): 2728. doi: 10.1097/WNP.0000000000000066.
9. McComas AJ. Invited review: motor unit estimation: methods, results, and present status. Muscle Nerve. 1991; 14(7): 585-97. doi: 10.1002/mus.880140702.

10. Eisen A, Karpati G, Carpenter S, et al. The motor unit profile of the rat soleus in experimental myopathy and reinnervation. Neurology. 1974; 24(9): 878-84 doi: 10.1212/wnl.24.9.878.

11. Arasaki K, Tamaki M, Hosoya $Y$, et al. Validity of electromyograms and tension as a means of motor unit number estimation. Muscle Nerve. 1997; 20(5): 552-60.

12. Li X, He W, Li C, et al. Motor unit number index examination in dominant and non-dominant hand muscles. Laterality. 2015; 20(6): 699-710. doi: 10.1080/1357650X.2015.1041971.

13. De Gennaro L, Cristiani R, Bertini $M$, et al. Handedness is mainly associated with an asymmetry of corticospinal excitability and not of transcallosal inhibition. Clin Neurophysiol. 2004; 115(6): 1305-12. doi: 10.1016/j.clinph.2004.01.014.

14. Triggs WJ, Calvanio R, Macdonell RA, et al. Physiological motor asymmetry in human handedness: evidence from transcranial magnetic stimulation. Brain Res. 1994; 636(2): 270-6. doi: 10.1016/0006-8993(94)91026-X.

15. Livingston SC, Goodkin HP, Ingersoll CD. The influence of gender, hand dominance, and upper extremity length on motor evoked potentials. J Clin Monit Comput. 2010; 24(6): 427-436. doi: 10.1007/s10877-010-9267-8.

16. Zhou P, Li X, Rymer WZ. Computing motor unit number index of the first dorsal interosseous muscle with two different contraction tasks. Med Eng Phys. 2012; 34(8): 1209-12. doi: 10.1016/j.medengphy.2012.06. 011.

17. Xu YS, Zheng JY, Zhang S, et al. [Comparison of multiple point stimulation and incremental stimulation in patients with amyotrophic lateral sclerosis]. Zhonghua Yi Xue Za Zhi. 2009; 89(43): 3051-4.

18. Cuturic $M$, Shamsnia $M$, Palliyath $S$. Lateral asymmetry of motor unit number estimate (MUNE). Electromyogr Clin Neurophysiol. 2005; 45(4): 233- 9.

Correspondence to Dr. Safa Dh. Abdul Muneem

E-mail: dr.saffadheaaalden@gmail.com Received Oct. $7^{\text {th }} 2018$

Accepted Jan. 23 2019 